EPJ Web of Conferences 101, 06010 (2015)

DOI: $10.1051 /$ epjconf/ 201510106010

(C) Owned by the authors, published by EDP Sciences, 2015

\title{
A planet orbiting an sdB star and an M dwarf in 2M 1938+4603
}

\author{
Adam Blokesz ${ }^{1, a}$, Andrzej Baran ${ }^{1}$, and Stanislaw Zola ${ }^{1,2}$ \\ 1 Pedagogical University of Cracow, ul. Podchorazych 2, 30-084 Cracow - Poland \\ 2 Astronomical Observatory of the Jagiellonian University, ul. Orla 171, 30-244 Cracow - Poland
}

\begin{abstract}
We present a preliminary analysis of the $2 \mathrm{M} 1938+4603$ star. It is an eclipsing binary system consisting of a primary sdB component and a secondary $\mathrm{M}$ dwarf. The photometric data are dominated by mutual eclipses and a very strong reflection effect. The primary has a fairly rich pulsation spectrum which can be used to study its interior. On the other hand, the pulsations affect the binary trend and vice versa what makes the analysis very difficult. Therefore, we attempted at proper modeling of the light variation due to eclipses and reflection and their removal from the data so it does not affect the Fourier analysis of stellar pulsations. We focus on mid-times of over 16000 primary and secondary minima, which were used to verify stability of the orbital period. The O-C diagram indicates possible parabolic and sinusoidal variations, commonly explained by period changes caused by evolution and a presence of a third body, respectively.
\end{abstract}

\section{Introduction}

We present a discovery of a tertiary component in $2 \mathrm{M} 1938+4603$, an eclipsing binary system consisting of a primary sdB star and a secondary $\mathrm{M}$ dwarf. The photometric data are dominated by mutual eclipses occurring every $0.1257653 \mathrm{~d}$. The object was observed by the Kepler spacecraft and the preliminary results were published by [4]. The sdB star is a rich, hybrid $\mathrm{p}$ and $\mathrm{g}$ modes pulsator. It pulsates in a very wide range of frequencies between 50 and $4531 \mu \mathrm{Hz}$. We modeled the binary trend [5] and removed it from the data so stellar oscillations can be touched. At this time we present our analysis of the orbital period variation.

\section{Orbital period analysis}

In total, there are over 16000 both primary and secondary eclipses. We used the Kwee and van Woerden's formalism [3] to evaluate mid-times of the eclipses and calculate the O-C diagram, which is shown in Fig. 1. The mid-times of both primary and secondary eclipses are plotted. Since the secondary eclipses are shallower the uncertainties of their mid-times measurements are larger. Both eclipses are in phase, suggesting that no apsidal motion exists in the system. The O-C diagram clearly shows that the orbital period is changing with time. To account for this change, we used parabolic and sinusoidal fits arriving at the following parameters:

$T_{0}=2455276.60843 \pm 0.00003$ BJD

$P=0.125765282 \pm 0.000000005$ days

$\dot{P}=(4.13 \pm 0.02) \times 10^{-11}$

$d=1.19 \pm 0.02 \mathrm{sec}$

$P^{\prime}=427 \pm 2$ days

a e-mail: ablokesz@gmail.com

This is an Open Access article distributed under the terms of the Creative Commons Attribution License 4.0, which permits unrestricted use, distribution, and reproduction in any medium, provided the original work is properly cited. 
$\phi=-2.43 \pm 0.04 \mathrm{rad}$

$$
P=T_{0}+P_{0} E+\frac{1}{2} \dot{P} P E^{2}+d \sin \left(\frac{2 \pi}{P^{\prime}} P E+\phi\right)
$$

The sinusoidal variation could be an indication of the periodic revolution of the barycenter of two main bodies around the tertiary component. The other secular change, represented by an opened-up parabola, can not be explained either by AML, magnetic braking or gravitational radiation, as they would result in period decrease. Assuming that the orbital inclinations of the tertiary component and the binary system are aligned, the mass of the companion would be 1.17 Jovian masses, and orbits the binaries at a distance of $0.78 \mathrm{AU}$. Since the sine curve fits the period changes relatively well, we assumed the orbit of the tertiary body to be circular.

\section{Rømer delay}

Because of the finite speed of light and unequal masses of the binary components a Rømer delay can be observed in this system [2]. The delay can be evaluated by means of the following formula $\Delta t_{R}=t_{s}-t_{p}-\frac{P}{2}$, where $t_{p}$ represents mid-times of primary eclipses and $t_{s}$ represents mid-times of secondary eclipses. The plot of the Rømer delay is shown in the top panel of Fig. 3. To check for a possible low amplitude variations we binned 20 consecutive points (middle panel of Fig. 4) and that binned diagram clearly shows a variation of the Rømer delay.

We applied a simultaneous parabolic and sinusoidal fits. A period of the Rømer delay variation equals $211 \pm 1$ days, and it is approximately twice shorter than the $P^{\prime}$. The amplitude of the sinusoid equals $1.18 \pm 0.01 \mathrm{sec}$ and this is the time during which light travels $0.5 \mathrm{R}_{\odot}$ distance. We offer no explanation for the Rømer delay variation.

A histogram presenting the Rømer delay is shown in Fig. 4. The Gaussian fit centers at $1.70 \pm 0.06$ sec, which is lower than the value derived by [1]. With the value of the Rømer delay derived in this work, $K_{1}=65.7 \mathrm{~km} / \mathrm{s}$ and inclination $=69^{\circ} .45$ [4], we recalculated the parameters of the binary: $q=0.308, M_{1}=0.26 \mathrm{M}_{\odot}, M_{2}=0.08 \mathrm{M}_{\odot}, a=0.81 \mathrm{R}_{\odot}, K_{2}=213 \mathrm{~km} / \mathrm{s}$

The mass of the primary component remains unexpectedly low for the surface gravity we derived from the spectroscopy. In addition it is half the canonical value widely accepted in other analyses. We guess that the Rømer delay is not properly measured, since it is indistinguishable from an ellipticity and therefore its evaluation may be strongly influenced. Our result of the simulations showing that the Rømer delay is hardly measurable in the Kepler short cadence data will be published elsewhere.

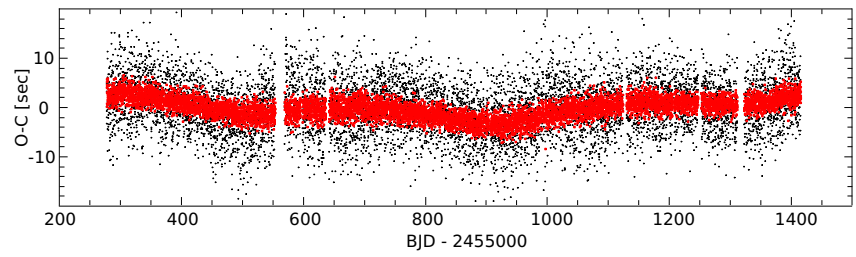

Fig. 1. The O-C diagram of $2 \mathrm{M} 1938+4603$; red dots represent primary eclipses, while secondary eclipses are shown with black dots.

This project was supported by Polish National Science Center grants No 2011/03/D/ST9/01914 and 2012/07/B/ST9/04432

\section{References}

1. Barlow, N. B., Wade, A. W., and Liss, E. S., Astrophys. J. 753, (2012) 101 


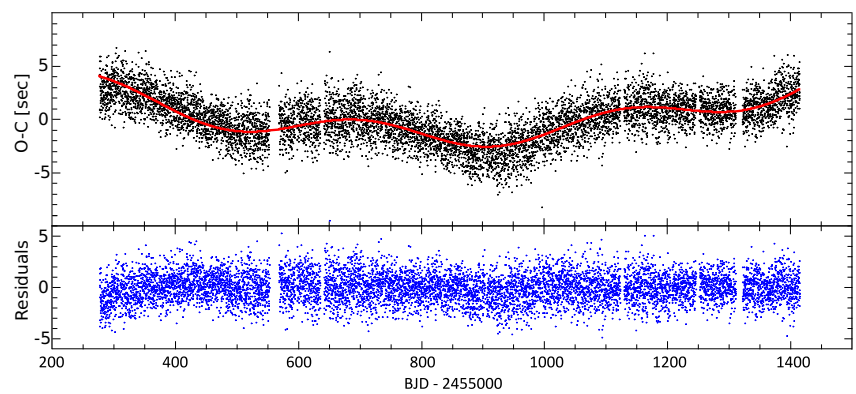

Fig. 2. Top panel: the O-C diagram with the parabolic+sinusoidal fits overplotted. Bottom panel: The residuals of the fit.

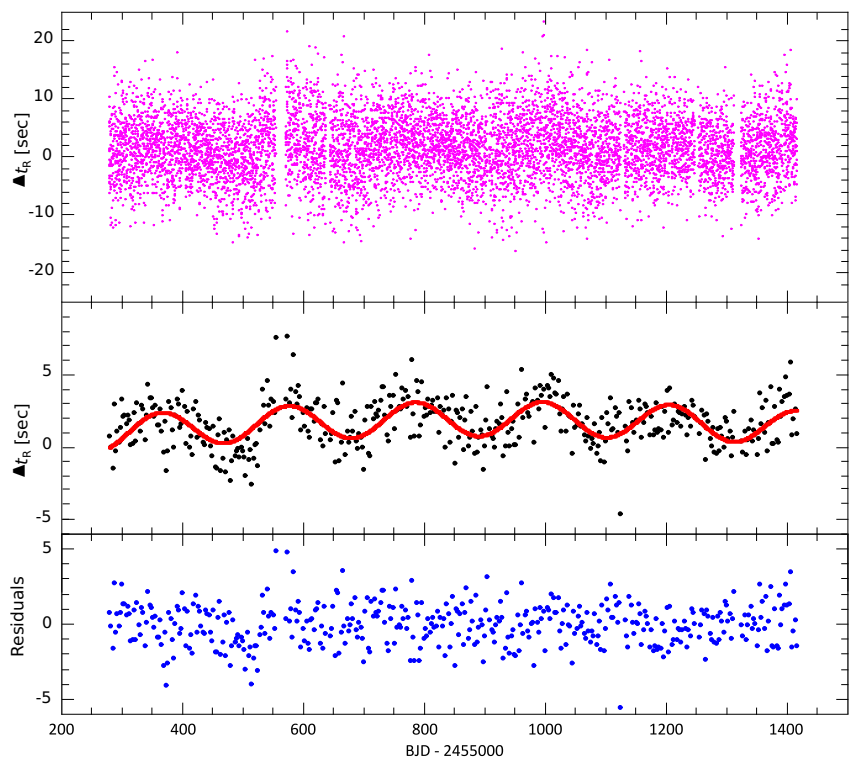

Fig. 3. The Rømer delay (top panel), 20-point binned Rømer delay with the parabolic+sinusoidal fits (middle panel) and the residuals of the fit (bottom panel).

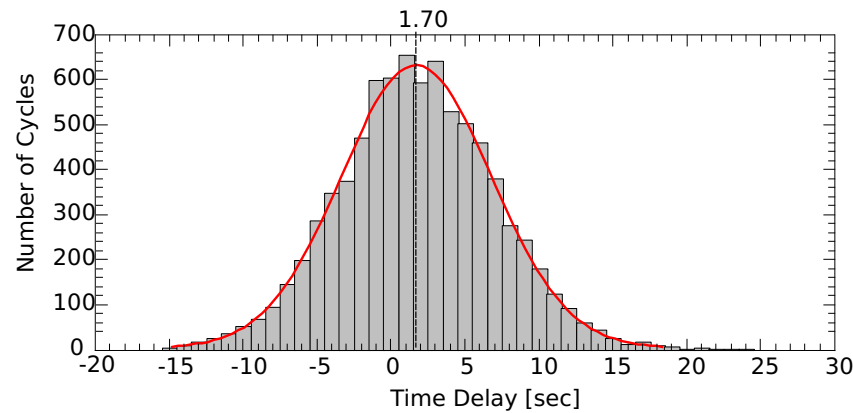

Fig. 4. A histogram of the Rømer delay with the Gaussian fit.

2. Kaplan D. K.:, Astrophys. J. 717, (2010) 108

3. Kwee, K. K., van Woerden, H., Bull. Astron. Inst. Netherlands 12, (1956) 327

4. Østensen, R. H., Green, E. M., Bloemen, S., et al, Mon. Not. R. Astron. Soc. 409, (2010) 147 
EPJ Web of Conferences

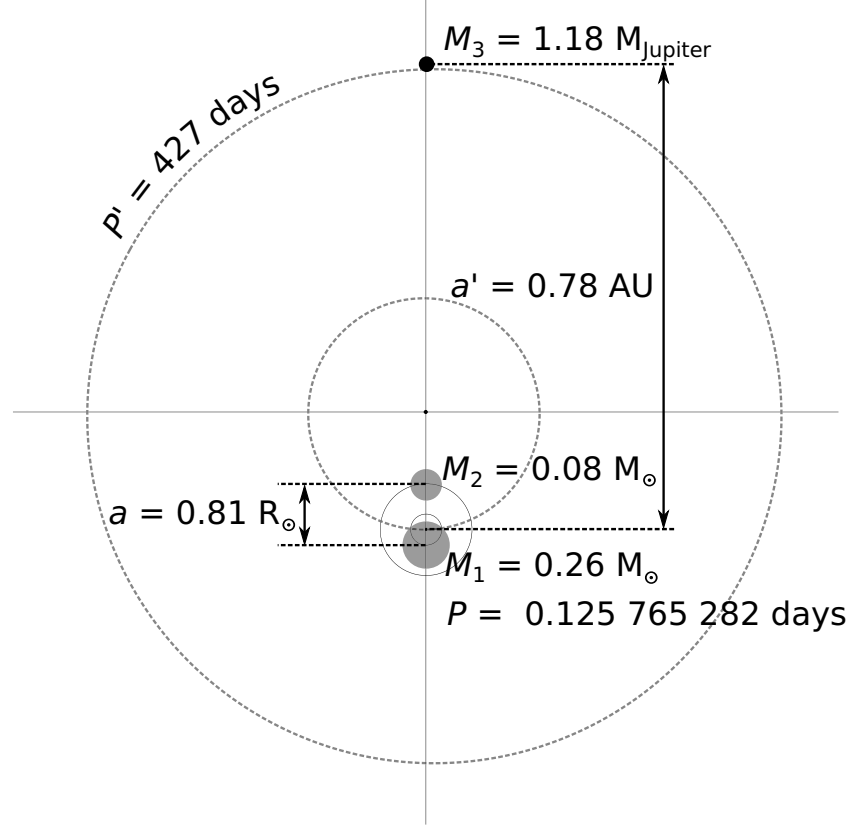

Fig. 5. Diagram representing the system.

5. Zola S., Baran A., Cent. Eur. Astrophys. Bull. 37, (2013) 227 